\title{
A Review on EEG Artifacts And Its Different Removal Technique
}

\author{
Chang Young Jung1), Sakala Satya Saikiran²)
}

\begin{abstract}
Electroencephalograms are the neurological signals which help in the study of various diseases. These are often contaminated with various artifacts. It is difficult to study and analysis of brain signals in the existence of these artifacts. EOG, ECG, motion and EMG are the common artifacts which cause disturbance to neurological signal. This review paper focuses on the artifact removal techniques with their features. Important parameters were taken into consideration while the study of various published papers. Strength and weakness of each paper are mentioned. This review of various papers is best of my knowledge.
\end{abstract}

Keywords: EEG, artifact, ocular, muscular, SNR, MSE, correlation coefficients

\section{Introduction}

Bio-signals or bioelectrical signals are the signals of electric or non-electric origin. These signals are produced by potential generated by a specialized cell system, tissues or organ system in the body.

There are some known bio-signals in the human body. These are:

\author{
Electrocardiogram (ECG) \\ Electromyogram (EMG) \\ Electroencephalogram (EEG) \\ Electrooculography (EOG) \\ Magnetoencephalogram (MEG) \\ Galvanic skin response (GSR) \\ Mechanomyogram (MMG)
}

Received(October 10, 2016), Review Result(1st: October 31, 2016, 2nd: December 2, 2016), Accepted(December 10, 2016)

${ }^{1}$ (Corresponding Author) Graduate School of Advanced Imaging \& Multimedia Chung-Ang University 84, Heukseok-ro, Dongjak-gu, Seoul, Korea email: mywood@naver.com

2) Department of Computer Science and Engineering, KL University email: Satyasaikiran9@gmail.com 
An Electroencephalogram (EEG) signal is a measurement of currents that flow during synaptic excitations of the dendrites of many pyramidal neurons in the cerebral cortex. When brain cells (neurons) are activated, the synaptic currents are produced within the dendrites. The current generates electrical field over the scalp measurable by EEG systems [1]. EEG is an essential element in the study and analysis of the neurological signals. These signals have the vital detail of the brain activity. These details are usedin the study of the diseases related to the brain like epilepsy.

Recording of brain signals are done with the help of different electrode system. There are two different ways of placing the electrode for the recording. First way is to place the electrode over the scalp. Second is to place them inside the scalp for the specific application. The recording for the EEG signals generally take over 20-30 minutes interval.The mostly used electrode system for the recording of the brain signal is 10-20 system. Figure 1 shows the placement of electrode for the recording of the electrode signal.
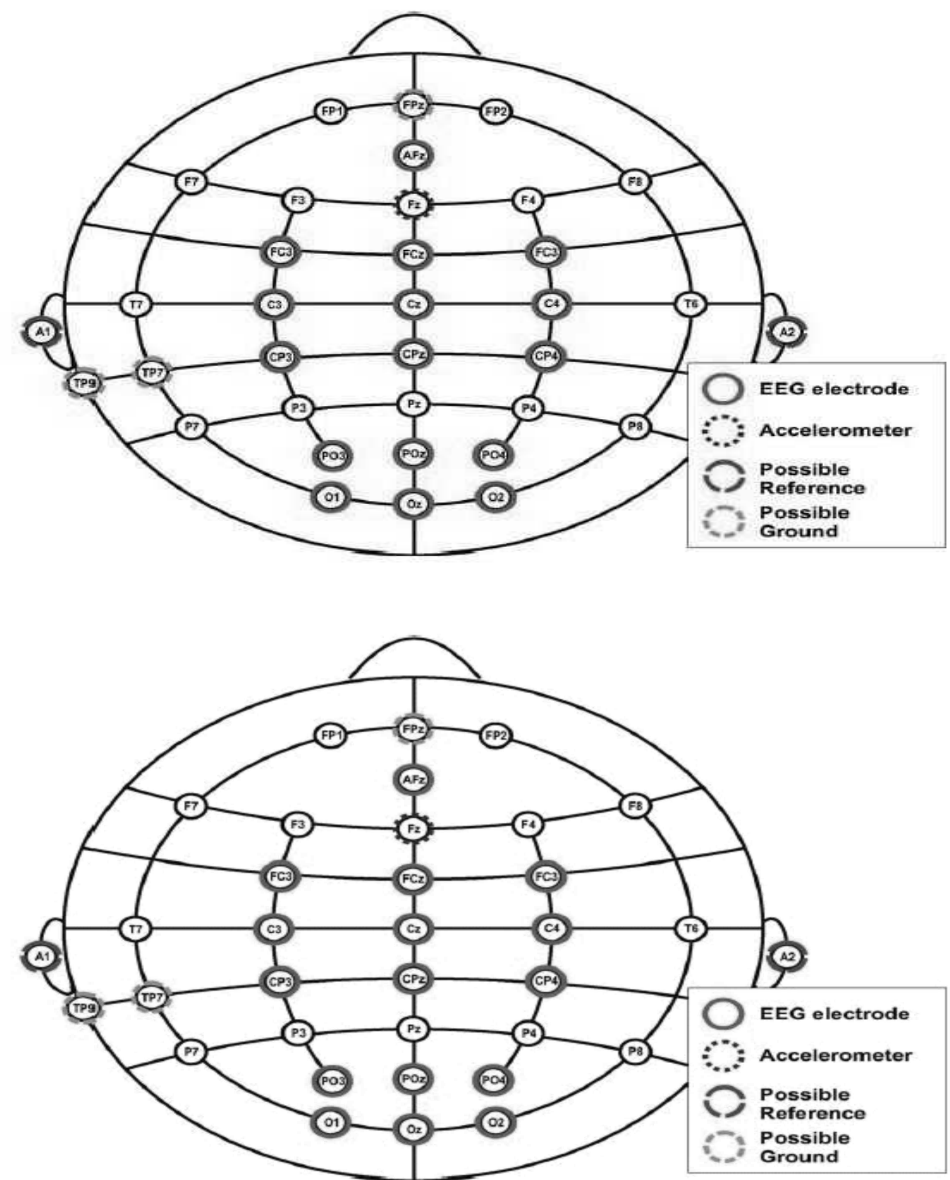
[Fig. 1] Electrode placement in EEG recording [1]

\subsection{Types of EEG signals}

EEG works on a very low frequency range. These waves are categorized as follows:

Delta waves: These waves range from $0-3.5 \mathrm{~Hz}$ frequency. These are generally broad, diffused shaped and may be bilateral with widespread. The subject's states are deep, trance, non-REM sleep and dreamless sleep.

Theta waves: These waves range from $4-7 \mathrm{~Hz}$ frequency. These are generally regional can have many lobes and may be lateralized or diffuse. The subject's states are instinctive, innovative, recall, drowsy and imaginary.

Alpha waves: These waves range from $8-13 \mathrm{~Hz}$ frequency. These are generally regional, involves full lobe. The subject's states are wakeful and resting.

Beta waves: These waves range from $14-40 \mathrm{~Hz}$ frequency. These are generally recorded from the parietal and frontal region of brain. These are further divided into various sub regions: Low beta, Mid-range beta, and High range beta.

Gamma waves: These waves range above $40 \mathrm{~Hz}$ frequency. These are much localized in nature. The subject's state is thinking.

These waves show the state of the person. We can use these waves as study and analysis of the brain activity and related problems with the help of different frequency components. Different frequency of brain waves is shown in figure 2.

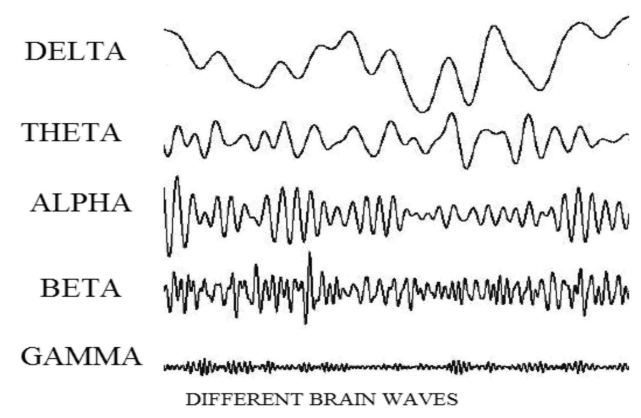

[Fig. 2] Different brain waves

\subsection{Artifacts}


A review on EEG artifacts and its different removal technique

The electroencephalogram is often contaminated with the noise. These noises may be physiological or non-physiological reason. In this review paper, we consider only physiological signal. These are:

1. Ocular artifact: Ocular artifact is the most common artifact during the measurement of the EEG signals. This artifact happens duepotential difference between cornea and retina. The movement of the eyelids or the blinking of eyescauses change in the potentials.

2. Muscle artifact:Muscle or EMG artifact is another cause noise in EEG signals. These are caused due to the movement of the frontalis and temporalis muscles.

3. Glossokinetic artifact: This artifact is caused due to the tongue. Tongue act as a dipole and movement of the tongue creates potential which spreads from fsrontal to occipital areas

4. ECG artifact: ECG artifact is due to the heart potential over the scalp. This type of artifact occurs in the case of the short and a wide neck person.

5. Pulse artifact: This artifact occurs when the electrode is placed over the pulsating vessel. This vessel can produce the artifact when the QRS complex occurs.

6. Respiration artifact: This artifact occurs when the movement of the body is due to the respiration. This causes the change in impedance of the electrodes which causes disturbance. This also happens when the patient is lying on electrode and continuously inhaling and exhaling.

7. Skin artifact: These artifacts occur when the impedance between the skin and the electrode change due to natural phenomena of the body.

\section{Artifact removal techniques}

During the recording of EEG the patientis asked to stay calm with no movement of any part. But it is impossible to stay stable during the recording. These artifacts combinewith the EEG signal which may give a wrong interpretation while analysing. So, the removal of these artifactsis necessary in order to get the correct information.

There are several artifact removal techniques. These techniques are applied to the raw EEG signal. Some of these techniques are given below

\subsection{Blind source separation (BSS)}

Blind source separation or BSS is a way to estimate different components from the mixtures 
of these signals. This method is called 'blind' because of the absence of any information except mixture. There are several techniques in blind source separation.

\subsubsection{Principle Component Analysis (PCA)}

Principal component analysis or PCA is a technique that uses statistical methods oforthogonal transformation which is used to change the observed component of uncorrelated variable to components of uncorrelated variables. These variables are known as called principal components. These principal components are less than the original variables.The principal components have the variance in decreasing order. The component observed first has highest variance followed by second and so on. Also,there should be orthogonality between these variables.

\subsubsection{Independent Component Analysis (ICA)}

Independent Component Analysis or ICA is a technique to separate the mixture of many signals into different additive independent component. These components are presumed of non-Gaussian and independent in nature. It works on the higher degree function. This is the advanced technique of Principal Component analysis.

Let us consider a signal is received with the mixture of different signals. This signal is represented as

$$
Y=A s Y=A s
$$

Here $Y$ is the received signal, is the source signals and is the different parameters depend upon different factors. Since receiving the original source signal from the mixture we have to compute the inverse of the matrix. This inverse of is represented as W.

$$
s=W Y s=W Y
$$

We have to compute this $\mathrm{W}$ with the help of different algorithm. Some of the algorithmsused in these papers are SCICA, AWICA, ORICA, JADE-ICA, jdICA, SOBI, fastICA etc.

\subsubsection{Canonical Correlation Analysis (CCA)}

Canonical Correlation Analysis or CCA is a technique for determination of linear relationship 
A review on EEG artifacts and its different removal technique

between two different sets of variables [9]. These associations are determined with the help of auto-covariance and cross-covariance of the variables. CCA works on the second order function. So, it is less complex as compared to Independent component analysis.

Let us consider a pair of sets of variables PP 
This $\mu \mu$

$\mathrm{Y}(\mathrm{t})$

$\mathrm{P}(\mathrm{t})$

$\mathrm{X}(\mathrm{t})$

$e_{u p}$

$e_{d n}$

$$
x_{i}[n]=x[n]+w_{i}[n] \quad w_{i}[n]
$$

$x_{i}[n]$

$\operatorname{IMF}_{\mathrm{k}}^{\mathrm{i}}[\mathrm{n}]$ 
A review on EEG artifacts and its different removal technique

the mode.

Assign $I M F_{K}^{I}[n]$ as kth mode of $\mathrm{x}[\mathrm{n}]$,

$$
I M F_{K}^{I}: I M F_{K}^{I}[n]=\frac{1}{N} I M F_{K}^{I}[n] I M F_{K}^{I}: I M F_{K}^{I}[n]=\frac{1}{N} I M F_{K}^{I}[n]
$$

\subsection{Wavelet Transform}

Wavelet transforms has an advantage over Fourier transforms is that it captures both frequency and time information which is limited to frequency information in the Fourier transform. This method also has advantage over BSS, EMD and EEMD. This method has faster execution time as compared to other methods. Wavelet transform can be expressed as

$$
F(a, b)=\int_{\infty}^{\infty} f(x) \Psi_{a . b}(x) d x \quad F(a, b)=\int_{\infty}^{\infty} f(x) \Psi_{a . b}(x) d x
$$

\subsubsection{Discrete Wavelet Transform}

Discrete Wavelets Transform (DWT) is the implementation of wavelet transform over discrete sets of wavelet scales. Wavelets in DWT are mutually orthogonal to each other. The scaling function is defined as

$\Phi(x)=\sum_{k=-\infty}^{\infty} a_{k} \Phi(S x-k) \Phi(x)=\sum_{k=-\infty}^{\infty} a_{k} \Phi(S x-k)$

Here $S$ is scaling factor and must be greater than 2 .

And orthogonality in the DWT can be defined as

$$
\int_{\infty}^{\infty} \Phi(x) \Phi(x+1) d x=\delta_{0.1} \int_{\infty}^{\infty} \Phi(x) \Phi(x+1) d x=\delta_{0.1}
$$

\subsubsection{Stationary Wavelet Transform}

Stationary Wavelet Transform or undecimatedwavelet transform is a technique of translation - invariance modification. Decimation of coefficient each level of transformation is done in SWT.

In classical DWT, the translated signal is not the same as compared toDWT of the original signal. So, classical DWT is not shift invariance. So, this problem of shift-invariance is done by removing the down-samplers and up-samplers in the DWT. Also up-sampling of the coefficients 
by a factor of the $2^{(j-1)} 2^{(j-1)}$

inputs

\section{there $\mathrm{s}$}


execution time of ICA with ICA-LMS, it is more. So, if considering the RMSE and correlation factor one can use the ICA-RLS, else in case of cost factor this method cannot be used. W. Y. Hsu et. al. [3] combined ICA and the correlation coefficient and used for the removalof the EOG from the EEG. After EOG removal features of the EEG is extracted with the DWT and amplitude modulation. Lastly, support vector machineis applied to grouping of the different EEG signals. Comparing the result with the EEG data containing artifacts, band power features and LDA classifier, it is a more accurate method.

[4-5] worked on different techniques to eliminate the eye blink artifact present in the EEG. J.W. Matiko et al. [4] used technique morphological component analysis is used along with STFT. The technique allows to present matrices based on eye blink and EEG separately. The Major benefit of this technique is need of less memory and computation time. Correlation coefficient obtained from the method is good. Considering these features in this method can be implemented for removal the EOG artifact. K.J. Huang et al. [5] used ORICA to eliminate the artifact. This method has advantagesof working in real time environment which was not present in the ICA method. ICA used to extract the artifactcontained data and then detection and removal of the artifact took place. Compared to that the execution time in ORICA is less without boundary missesand the correlation coefficient is high. So, ORICA is preferred over the ICA method of eye blink artifact removal as it is a real time algorithmwith feasibility and reliability.

M. Betta et al. [6] proposed an algorithm to detect REM sleep and then eliminationof the ocular artifact from the EEG data. This algorithm uses the integration of the Wavelet transform with adaptive filtering. Comparing this algorithm with adaptive filtering alone, it shows better accuracy. This method is helpful in determining the actual noise of EEG data due to REM.

M. H. Soomro et al. [7, 8, and 9] proposed different algorithm for the removal of the eye blink artifact from the EEG data. [8] In first method EMD is usedwith the ICA. In this method the SAR and average correlation coefficient was better compared to fast ICA. But when compared with the FastICA algorithm, the execution time for the processing of the artifact removal was more. Also the algorithm was not feasible with the real time artifact removal. In further work, a new method proposed by the author. [7] In this, a combination of EMD and CCA is used to remove the eye blink artifacts. The result of EMD-CCA compared with ICA, CCA and EMD-fastICA in terms of elapsed time, SAR and average correlation coefficient. In case of average correlation coefficient and SAR this algorithm outperformed all other algorithm. But in case of execution time it is more than CCA. Considering the accuracy, this method can be used in the real time application. [9] In further work, author compared two BSS algorithm 
i.e. fastICA and CCA. Both of these algorithms performed better in the elimination of artifact for the three channels EEG data. In the execution time the CCA is better than ICA. Also, both CCA and fastICA should have higher accuracy. So EMD-CCA was the better comparing all three algorithms.

[10-11]proposed different techniques to eliminate the eye blink artifact. N. Mourad et al. [10] used method of Empirical mode decomposition to remove the artifact. Reference signal and dictionary matrices are createdfor eye blink artifact using EMD. In further step the signal with the reference signal is matched and the required EEG data are given. Comparing EMD with Blind source separation method the result was better in the case of the EMD. P.A. Bizopolous et al. [11] used Ensemble empirical mode decomposition algorithm for artifact removal. A NormalizedCorrelation Coefficient prepared based on the previouslydetermined blinking artifact library. The proposed method eliminates the artifact based on the data present in the library. The proposed method shows better average SER and RMSE.

R. Mahajan et al. [12-13] proposed different method to eliminate the Eye blink artifact removal. In first method Enhanced entropy based Wavelet ICA is used. [12] The comparison of proposed method is done with the Zeroing-ICA and wavelet ICA and found that this method is numerically efficient than both in terms of SNR, mutual information and computational time with higher accuracy. Also, no reference signal is needed for the elimination of artifact from the EEG. [13] In the second methodalong with wavelet ICA algorithm modified multiscale entropy, entropy and courtesies are used to identify and eliminate the data. This suggested method is matched with the wICA and Zeroing-ICA and result show better performance in case of SNR, correlation coefficient and spectral coherence.

S. Khatun et al. [14]worked on different approach of eliminating eye blink artifact. These approaches were compared to each other. These approaches consist of DWT and SWT with different basis function and statistical thresholding and universal thresholding. Considering the correlation factor DWT-UT is the best, while SWT-ST is best in case of NMSE and time, frequencyanalysis and DWT-ST best in execution time.

Q. Zhao et al. [15] used method DWT algorithm with adaptive predictor to remove the ocularartifact from EEG signal. This method is compared to ICA and WPT, DWT and ANC and found that it was better interms of RMSE and correlation coefficient. In case accuracy and efficiency this method is not good enough. C. Zhao et al. [16] used method of wavelet enhanced CCA is used to remove the EOG artifact. This method compared to CCA, ICA and wICA and observation shows that while removing ocular artifact there is minimum loss of information. This method performed on the real EEG data and it removed EOG artifact with 
few signal change. C. Burger et al. [17] used method consists of ICA and a wavelet neural network algorithm for ocular artifact removal. When this method is compared to ICA method the results were better in denoising. Independent component from the ICAis applied to WNN result in correcting the ocular artifact from the EEG Signal with little loss.

E. Lyzhko et al. [18] workedon the ocular artifact removal from the Trans cranial magnetic simulation and the EEG combination. In this method three algorithms of ICA are compared. TDSEP algorithm of ICA is better than runICA and fastICA. G. Wang et al. [19]proposed method to eliminate Ocular artifact from the EEG signal from multichannel data using independent component analysis and multivariate empirical mode decomposition. This method removed the ocular artifact with little loss. When ICA-MEMD compared with AWICA and SSA-EMD then result were better in SNR and MSE in ICA-MEMD. H.A.T Nguyen et al. [20] used wavelet transformsand neural network for the removal of EOG signal from EEG. WNN identifies the characteristics ofthe reference EEG data. After that there will be no need of reference signal while removing the Ocular artifact from the EEG signal. This method is compared with ICA and resultsshow that WNN is more efficient than the ICA in very noisy EEG data.

$[21,25]$ worked on a technique to eliminate the EOG and EMG artifact from the EEG signal. M.K. Mowla et al. [21] used CCA-SWT to remove EMG artifact while SOBI-SWT to eliminate the EOG artifact. The method is compared to the BSS-SCD and the correlation coefficient is better in both the case of CCA-SWT and SOBI-SWT. J. Hu et al. [25] used algorithm of ANFIS-FLNN to make a filter that removes EOG and EMG artifact from EEG. This method result is further compared to ANFIS, RBF-ANFIS and adaptive FL-BPNN filters. The result of this method outperformed other artifact removal method.

R. Patel et al. [22] used ensemble empirical mode decomposition and principal component analysis to eliminate the ocular artifact from the EEG data. EMD is helpful for single channel data, whereasPCA and ICA can be used if the EEG channels are large. This method relies on the proper detection of the EEG artifact.

S. Kanoga et al. [23] used complete ensemble empirical mode decomposition (CEEMD) and independent component analysis (ICA) are used to eliminate the eye blink artifact. CEEMD-ICA is compared to the wICA, EMD-ICA and EEMD-ICA. Performance of CEEMD-ICA is better when comparing through SNR. W. D. Chang et al. [24] applied MSDW method to classify the eye blink artifact. This experiment was performed on the 24 subjects. Theresults were compared with the conventional method and result shows that the MSDW is better at detecting the error from the EEG data. 
S. Ge et al. [26] used Undetermined Blind Source Separation to eliminate the ocular artifact present in the signal. In this FOOBI algorithm of ICA is used to separate the different component. This method is compared with the other method of ICA. This method gave better accuracy than different methods of ICA. C. A. Majmdar et al. [27] proposed algorithm uses the DWT method to detect and denoisethe ocular artifact from the single channel EEG. This method was compared to SWT. DWT showed better performance in different parameter. Also the execution speed was faster than SWT. It is better to use DWT when removing the artifact from wavelet. This method can also be used for the multichannel EEG data.

P.N. Jadhav et al. [28]worked on the technique of elimination of EMG and EOG artifact from EEG data. In this method fastICA along with wavelet transform is used to remove the artifact. Firstly fastICA is applied to the raw EEG data, thenmuscle artifact is removed and lastly ocular artifact is removed from the EEG data. The experiment is done on 20 subjects. The correlation coefficient and regression show the acceptable performance of this technique.

D. Safieddine et al. and C. Teng et al. worked on the removal of EMG artifact of the EEG signal. [29] In this method comparison had done between EMD, ICA, CCA and WT algorithms. For the low SNR signal EMD should be preferred but ICA-EMD will give better result. For high SNR signal the preference should be given to 2T-EMD or CoM2. The complexity was least in case of DWT and CCA. [30] In this method MEMD is used. In first step multivariate IMF is generated to then power spectra is calculated for each MIMF. Since the power spectra of EMG and EEG are different. The EMG signal power spectrum is removed by assigning zero. Thus we can remove the artifact. This technique is matched with ICA techniques and the result come with better SNR and MSE.

M. Anastasiadou et al. [31, 32] suggested two methods to eliminate an EMG artifact from the EEG signal. At first method CCA is applied to remove the muscle artifact. [31] This experiment was performed on three patients. [32] At second method CCA and WT is applied to remove the EMG artifact. In this method specificity and sensitivity was computed. Results of patients were found satisfactory. These both techniques provide technique to remove artifact of EEG signal of the patient of epilepsy in real time. S.H. Sardouie et al. [33] worked on the elimination of muscle artifact from the EEG in the paediatric patient. In this technique JDICA is used to eliminatethe artifact. This method uses the fourth order commulant to separate the independent component. This method is compared with fastICA, robustICA and DEL algorithm of ICA and found thatit worked better if the number of electrode is less.

$X$. Chen et al. [34-35] proposed two approaches for the elimination of EMG artifact from the single channel EEG signal. [34] In first method EEMD-MCCA is used to remove the artifact. 
[35] In further method EEMD and JBSS technique were used to eliminate the artifact signal form the EEG data. First combination was taken as EEMD-IVA and second EEMD-CCA. EEMD-CCA performed better than EEMD-IVA with better efficiency. Both techniques can work in more number of channels but should be less. These both techniques are helpful in real life application like ambulatory service.

K.T. Sweeney et al. [36] proposed method to eliminate the muscle artifact form the EEG. The EEMD-ICA is used in this for the elimination of the EMG artifact from the raw signal. The correlation coefficient and SNR are taken into account. This is the simple and reliable approach to eliminate the EMG artifact from the EEG. In further work [37] proposed method to eliminate the automatic artifact detection and removal from EEG signals. These methods consist of EEMD-CCA algorithm. This method calculates the SNR, MSE and correlation coefficient and then compared with the method of EEMD-ICA. Results are better in the case of the EEMD-CCA.

M. K. Islam et al. worked on different algorithms to automatically detect and eliminate the artifact from the raw EEG. [38] In this method SWT is used for the elimination of artifact. This method is further compared with the ICA, wICA, wCCA, EMD-ICA and EMD-CCA. Results are better in the case of SWT. [39]In this method WT is used for the elimination of artifact. There is an increase in the accuracy in this case byremoving the artifact from the EEG dataset. This algorithm tested on the real dataset and there also the results were good.

I. Daly et al. [40] worked on automatic detection and elimination of artifact method from EEG. This method is working on combination of ICA, wavelet decomposition and thresholding known as FORCe algorithm. Thismethod performed on 13 subjects with cerebral palsy and 3 healthy subjects. The result is then compared with the LAMIC and FASTER algorithm and its performance was good in comparison to them. This method can remove ECG, EOG, EMG, and blink artifact with 16 channel data.

J.A. Jiang et al. [41] worked on method for the ECG artifact removal in EEG signal. Wavelet transform has been used for the removalof the artifact. The result shows the better artifact detection and removal in the experiment.

\section{Conclusion}

The authorshave worked on EOG, EMG, ECG and motion. Some of them have worked on automatic detection and removal of artifacts. BSS, WT, EMD, Neural networks and other 
techniques have been applied. Papers have taken different parameters like correlation coefficient, MSE, SNR, execution speed, complexity etc. These parameters are helpful in analysing the technique. Some of technique like ICA gives good result but have the high complexity and low speed. Mostly BSS has been used for the removal of the techniques. In future there may be a combination of these techniquesmay be used to increase the efficiency and speed of the removal of the artifacts.

\section{References}

[1] coep.vlab.co.in, To Simulate Electroencephalogram Signal, (2011).

[2] C. G. Mosquera and A. N. Vazquez, Automatic Removal of Ocular Artefacts Using Adaptive Filtering and Independent Component Analysis for Electroencephalogram Data, Signal Process IET, (2012), Vol.6, No.2, pp.99-106.

[3] W. Y. Hsu, C. H. Lin, H. J. Hsu, P. H. Chen, and I. R. Chen , Wavelet-Based Envelope Features with Automatic EOG Artifact Removal: Application to Single-Trial EEG Data, Expert Systems with Applications, (2012), Vol.39, pp.2743-2749.

[4] J. W. Matiko, S. Beeby, and J. Tudor, Real Time Eye Blink Noise Removal from EEG Signals Using Morphological Component Analysis, Engineering in Medicine and Biology Society (EMBC), (2013), pp. 13-16.

[5] K. J. Huang,J. C. Liao, W. Y. Shih, C. W. Feng, J. C. Chang, C. C. Chou, and W. C. Fang, "A Realtime Processing flow for ICA based EEG Acquisition system with Eyeblink Artifact Elimination," Signal Processing Systems (SiPS), pp. 237-240, (2013).

[6] M. Betta, A. Gemignani, A. Landi, M. Laurino, P. Piaggi, and D. Menicucci, Detection and Removal of Ocular Artifacts from EEG signals for an Automated REM sleep analysis, Engineering in Medicine and Biology Society (EMBC), (2013), pp.5079-5082.

[7] M. H. Soomro, N. Badruddin, M. Z. Yusoff, and M. A. Jatoi, Automatic Eye-Blink Artifact Removal Method Based on EMD-CCA, Complex Medical Engineering (CME), (2013), pp.186-190.

[8] M. H. Soomro, N. Badruddin, M. Z. Yusoff, and A. S. Malik, A Method for Automatic Removal of Eye Blink Artifacts from EEG Based on EMD-ICA, Signal Processing and its Applications (CSPA), (2013), pp.129-134.

[9] M. H. Soomro, N. Badruddin, and M. Z. Yusoff, Comparison of Blind Source Separation Methods for Removal of Eye Blink Artifacts from EEG, Intelligent and Advanced Systems (ICIAS), (2014), pp.1-6.

[10] N. Mourad and R. K. Niazy, Automatic Correction of Eye Blink Artifact in Single Channel Recording using EMD and OMP, Signal Processing Conference (EUSIPCO), 2013 Proceedings of the 21st European, (2013), pp.1-5.

[11] P. A. Bizopoulos, T. Al-Ani, D. G. Tsalikakis, A. T. Tzallas, D. D. Koutsouris, and D. I. Fotiadis, An 
Automatic Electroencephalography Blinking Artefact Detection and Removal Method Based on Template Matching and Ensemble Empirical Mode Decomposition, Engineering in Medicine and Biology Society (EMBC), (2013), pp.5853-5856.

[12] R. Mahajan and B. I. Morshed, "Sample Entropy Enhanced Wavelet-ICA Denoising Technique for Eye Blink Artifact Removal from Scalp EEG Dataset," Neural Engineering (NER), (2013), pp.1394-1397.

[13] R. Mahajan and B. I. Morshed, "Unsupervised Eye Blink Artifact Denoising of EEG Data with Modified Multiscale Sample Entropy, Kurtosis, and Wavelet-ICA," Biomedical and Health Informatics, (2014), Vol.19, No.1, pp.158-165.

[14] S. Khatun, R. Mahajan, and B. I. Morshed, Comparative Analysis of Wavelet Based Approaches for Reliable Removal of Ocular Artifacts from Single Channel EEG, Electro/Information Technology (EIT), (2015), pp.335-340.

[15] Q. Zhao, B. Hu, Y. Shi, Y. Li, P. Moore, M. Sun, and H. Peng, Automatic Identification and Removal of Ocular Artifacts in EEG-Improved Adaptive Predictor Filtering for Portable Applications, NanoBioscience, (2014), Vol.13, No.2, pp.109-118.

[16] C. Zhao and T. Qiu, An Automatic Ocular Artifacts Removal Method Based On Wavelet-Enhanced Canonical Correlation Analysis, Engineering in Medicine and Biology Society (EMBC), (2015), pp.4191-4194.

[17] C. Burger and D. J. van den Heever, Removal of EOG Artefacts by Combining Wavelet Neural Network and Independent Component Analysis, Biomedical Signal Processing and Control, (2015), Vol.15, pp.67-79.

[18] E. Lyzhko, L. Hamid, S. Makhortykh, V. Moliadze, and M. Siniatchkin, Comparison of Three ICA Algorithms for Ocular Artifact Removal from TMS-EEG Recordings, Engineering in Medicine and Biology Society (EMBC), (2015), pp.1926-1929.

[19] G. Wang, C. Teng, K. Li, Z. Zhang, and X. Yan, The Removal of EOG Artifacts from EEG Signals Using Independent Component Analysis and Multivariate Empirical Mode Decomposition, Biomedical and Health Informatics, (2015), Vol.99, pp.1-7.

[20] H. A. T. Nguyen, J. Musson, F. Li, W. Wang, G. Zhang, R. Xu, C. Richey, T. Schnell, F. D. McKenzie, and J. Li, EOG Artifact Removal Using a Wavelet Neural Network, Neurocomputing, (2015), Vol.97, pp.374-389.

[21] M. R. Mowla, S. C. Ng, M. S. A. Zilany, and R. Paramesran, Artifacts-Matched Blind Source Separation and Wavelet Transform for Multichannel EEG Denoising, Biomedical Signal Processing and Control, (2015), Vol.22, pp.111-118.

[22] R. Patel, S. Sengottuvel, M. P. Janawadkar, K. Gireesan, T. S. Radhakrishnan and N. Mariyappa, Ocular Artifact Suppression from EEG Using Ensemble Empirical Mode Decomposition with Principal Component Analysis, Computers and Electrical Engineering, (2015).

[23] S. Kanoga and Y. Mitsukura, Eye Blink Artifact Rejection in Single-Channel Electroencephalographic Signals by Complete Ensemble Empirical Mode Decomposition and Independent Component Analysis, Engineering in Medicine and Biology Society (EMBC), (2015), pp.121-124.

[24] W. D. Chang, H. S. Cha, K. Kim, and C. H. Im, Detection of Eye Blink Artifacts from Single Prefrontal Channel Electroencephalogram, Comput. Methods Programs Biomed, (2015). 
[25] J. Hu, C. Wang, M. Wu, Y. Du, Y. He, and J. She, Removal of EOG and EMG Artifacts from EEG Using Combination of Functional Link Neural Network and Adaptive Neural Fuzzy Inference System, Neurocomputing, (2015), Vol.151, No.1, pp.278-287.

[26] S. Ge, M. Han and X. Hong, A Fully Automatic Ocular Artifact Removal from EEG Based on Fourth-order Tensor Method, Biomed. Eng. Lett., (2014), Vol.4, pp.55-63.

[27] C. A. Majmudar, R. Mahajan, and B. I. Morshed, Real-Time Hybrid Ocular Artifact Detection and Removal for Single Channel EEG, Electro/Information Technology (EIT), (2015), pp.330-334.

[28] P. N. Jadhav, D. Shanamugan, A. Chourasia, A. R. Ghole, A. Acharyya, and G. Naik, Automated Detection and Correction of Eye Blink and Muscular Artefacts in EEG Signal for Analysis of Autism Spectrum Disorder, Engineering in Medicine and Biology Society (EMBC), (2014), pp.1881-1884.

[29] D. Safieddine, A. Kachenoura, L. Albera, G. Birot, A. Karfoul, A. Pasnicu, A. Biraben, F. Wendling, L. Senhadji, and I. Merlet, Removal of Muscle Artifact from EEG Data: Comparison Between Stochastic (ICA and CCA) and Deterministic (EMD and Wavelet-Based) Approaches, EURASIP Journal on Advances in Signal Processing, (2012), Vol.127, pp.1-15.

[30] C. Teng, Y. Zhang, and G. Wang, The Removal of EMG Artifact from EEG Signals by the Multivariate Empirical Mode Decomposition, Signal Processing, Communications and Computing (ICSPCC), (2014), pp.873-876.

[31] M. Anastasiadou, A. Hadjipapas, M. Christodoulakis, E. S. Papathanasiou, S. S. Papacostas, and G. D. Mitsis, Detection and Removal of Muscle artifacts from Scalp EEG Recordings in Patients with Epilepsy, Bioinformatics and Bioengineering (BIBE), (2014), pp. 291-296.

[32] M. Anastasiadou, M. Christodoulakis, E. S. Papathanasiou, S. S. Papacostas and G. D. Mitsis, Automatic Detection and Removal of Muscle artifacts from Scalp EEG Recordings in Patients with Epilepsy, Engineering in Medicine and Biology Society (EMBC), (2015), pp.1946-1950.

[33] S. H. Sardouie, L. Albera,M. B. Shamsollahi and I. Merlet, An Efficient Jacobi-Like Deflationary ICA Algorithm: Application to EEG Denoising, Signal Processing Letters, (2015), Vol.22, No.8, pp.1198-1202.

[34] X. Chen, A. Liu, H. Peng, and R. K. Ward, A Preliminary Study of Muscular Artifact Cancellation in Single-Channel EEG, Sensors, (2014), Vol.14, No.10, pp.18370-18389.

[35] X. Chen, C. He, and H. Peng, Removal of Muscle Artifacts from Single-Channel EEG Based on Ensemble Empirical Mode Decomposition and Multiset Canonical Correlation Analysis, Journal of Applied Mathematics, (2014), Vol.2014, No.261347, pp.1-10.

[36] K. T. Sweeney, S. F. McLoone, and T. E. Ward, The Use of Ensemble Empirical Mode Decomposition with Canonical Correlation Analysis as a Novel Artifact Removal Technique, Biomedical Engineering, (2013), Vol.60, No.1, pp.97-105.

[37] K. T. Sweeney, H. Ayaz, T. E. Ward, M. Izzetoglu, S. F. McLoone, and B. Onaral, A Methodology for Validating Artifact Removal Techniques for Physiological Signals, Information Technology in Biomedicine, (2012), Vol.16, No.5, pp.918-926.

[38] M. K. Islam, A. Rastegarnia, A. T. Nguyen, and Z. Yang, Artifact Characterization and Removal for In-Vivo Neural Recording, Journal of Neuroscience Methods, (2014), Vol.226, pp.110-123. 
[39] M. K. Islam, A. Rastegarnia, and Z. Yang, A Wavelet-Based Artifact Reduction from Scalp EEG for Epileptic Seizure Detection, Biomedical and Health Informatics, (2015), Vol.99, pp.1-12.

[40] I. Daly, R. Scherer, M. Billinger, and G. M. Putz, FORCe: Fully Online and Automated Artifact Removal for Brain-Computer Interfacing, Neural Systems and Rehabilitation Engineering, (2015), Vol.23, No.5, pp.725-736.

[41] J. A. Jiang, C. F. Chao, M. J. Chiu, R. G. Lee, C. L. Tseng, and R. Lin, An Automatic Analysis Method for Detecting and Eliminating ECG Artifacts in EEG, Computers in Biology and Medicine, (2007), Vol.37, pp.1660-16710. 